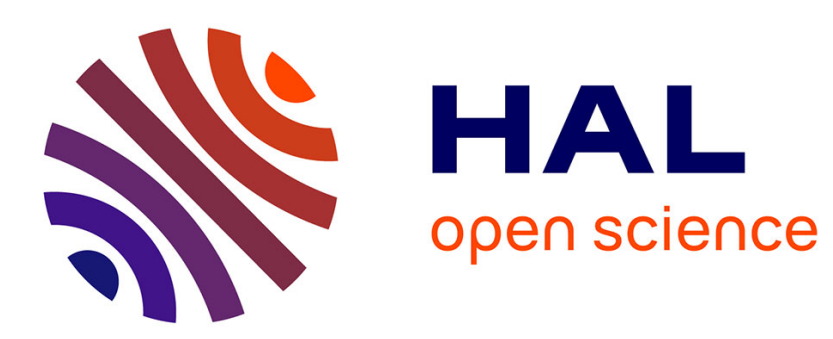

\title{
Croissance osseuse au cours des périodes foetale et néonatale: rôle du peptide apparenté à l'hormone parathyroïdienne
}

\author{
J.-P. Barlet, M.-J. Davicco, Véronique Coxam
}

\section{To cite this version:}

J.-P. Barlet, M.-J. Davicco, Véronique Coxam. Croissance osseuse au cours des périodes foetale et néonatale: rôle du peptide apparenté à l'hormone parathyroïdienne. Productions Animales, 1995, 8 (3), pp.177-187. hal-00896114

\section{HAL Id: hal-00896114 https://hal.science/hal-00896114}

Submitted on 1 Jan 1995

HAL is a multi-disciplinary open access archive for the deposit and dissemination of scientific research documents, whether they are published or not. The documents may come from teaching and research institutions in France or abroad, or from public or private research centers.
L'archive ouverte pluridisciplinaire HAL, est destinée au dépôt et à la diffusion de documents scientifiques de niveau recherche, publiés ou non, émanant des établissements d'enseignement et de recherche français ou étrangers, des laboratoires publics ou privés. 
INRA Prod. Anim., 1995, 8 (3), 177-187

\section{J.-P. BARLET, M.-J. DAVICCO, V. COXAM}

INRA Laboratoire Croissance et Métabolismes des Herbivores

Theix 63122 Saint Genès Champanelle

\section{Croissance osseuse au cours des périodes fotale et néonatale : rôle du peptide apparenté à l'hormone parathyroïdienne}

La croissance osseuse fœtale et néonatale conditionne en grande partie le format adulte et, ainsi, les potentialités ultérieures d'un animal. Sa régulation pose encore de nombreux problèmes, en particulier chez les ruminants domestiques. Quels sont les facteurs endocriniens contrôlant les transferts placentaires de calcium et de phosphore? Comment est régulée l'absorption intestinale des minéraux du lait chez le nouveau-né ? La découverte récente (1988) du peptide apparenté à l'hormone parathyroïdienne, responsable de l'hypercalcémie associée à certaines formes de cancers, et la mise en évidence de certains de ses rôles physiologiques permettent de répondre à bon nombre de ces questions.

Chez les mammifères, la régulation endocrinienne des métabolismes phospho-calcique et osseux est essentiellement assurée par les hormones calciotropes. Le 1,25-dihydroxycholécalciférol (1,25-(OH)2 D3), métabolite actif de la vitamine D3, stimule l'absorption intestinale du calcium et du phosphore, et la minéralisation osseuse. La calcitonine (CT), peptide sécrété par les cellules parafolliculaires thyroïdiennes, inhibe la résorption osseuse et module les transferts calciques placentaires,

\footnotetext{
Résumé

Le peptide apparenté à l'hormone parathyroïdienne (PTHrP) joue un rôle important dans la régulation de la croissance osseuse fotale et néonatale. Chez la femelle gestante et/ou allaitante, l'hormone sécrétée par les parathyroïdes fœtales, les membranes placentaires et/ou la glande mammaire en lactation, stimule les transferts calciques placentaires, module la tonicité utérine en fonction de la taille du (ou des) foetus, contrôle le débit artériel mammaire et la teneur du lait en calcium et en phosphore. Chez le nouveau-né, le PTHrP apporté par le lait pourrait intervenir dans la régulation de la motricité intestinale et de l'absorption intestinale du calcium et du phosphore.
}

évitant ainsi une déminéralisation excessive du squelette de la femelle gestante et/ou allaitante. L'inhibition de la résorption osseuse induite par la CT résulte de l'induction de la rétraction et de la quiescence ostéoclastiques (Weryha et Leclère 1995). L'hormone parathyroïdienne (PTH), antagoniste de la CT au niveau osseux, agit en synergie avec celle-ci au niveau rénal : l'une et l'autre induisent une phosphaturie en diminuant la réabsorption tubulaire des phosphates. Si la 1,25 -(OH)2 D3 peut stimuler les transferts calciques placentaires (Ross et al 1980) et la sécrétion mammaire de calcium (Roux et al 1979), les hormones peptidiques (PTH et CT) ne semblent pas jouer un rôle important au niveau mammaire où l'une et l'autre s'avèrent incapables de modifier la composition minérale du lait (Munson 1955, Barlet 1974). Chez l'animal, la parathyroïdectomie entraîne une baisse de la calcémie et une élévation de la phosphatémie. Cette dernière résulte des effets rénaux de l'hormone, l'hypocalcémie provenant d'un ralentissement de l'activité ostéoclastique (Parsons 1976). 
Un syndrome particulier, désigné sous le nom d'hypercalcémie humorale maligne, est associée à certains cancers humains ou canins. Chez les patients humains ou animaux, les paramètres biochimiques sanguins évoquent une hyperparathyroïdie : hypercalcémie, hypophosphatémie, augmentation de la résorption osseuse et de l'excrétion urinaire d'AMP cyclique (AMPc). Néanmoins, chez ces individus, les concentrations plasmatiques de PTH, évaluées par dosage radioimmunologique sont faibles et l'étude cytophysiologique des parathyroïdes d'animaux atteints révèle leur inactivité (Rosol et Capen 1988). Ceci indique qu'un facteur d'origine extraparathyroïdienne, mais ayant des effets biologiques analogues à ceux de PTH, dont il diffère du point de vue immunologique, est présent chez ces patients.

Un peptide ayant les mêmes effets biologiques que $\mathrm{PTH}$ a effectivement été isolé de ces tumeurs (Moseley et al 1987, Broadus et al 1988). Il a été appelé «PTH-related Peptide (PTHrP).

\section{1 / Biochimie du peptide apparenté à l'hormone parathyroïdienne}

Le gène responsable de la synthèse du PTHrP a été isolé et cloné. Chez l'homme, il est localisé sur le bras court du chromosome 11. Plusieurs formes d'ARNm résultant d'épissages alternatifs ont été décrites et codent pour trois séquences de PTHrP différant par leur extrémité C-terminale : PTHrP (1-139), PTHrP (1-141), et PTHrP (1-173) (figure 1), la séquence (1-139) étant la même dans les trois formes. Actuellement, seules les séquences aminées du PTHrP de l'Homme, de la souris, du rat et du poulet sont connues.
Huit des treize premiers acides aminés $\mathrm{N}$ terminaux du PTHrP sont identiques à ceux de la même séquence de la PTH. Cette similitude explique la capacité de ces deux molécules à réagir avec le même récepteur dont le gène a été cloné et la structure déterminée (Jüppner et al 1991). Les séquences synthétiques humaines (1-34), (1-141) et (1-173) possèdent la même activité biologique que la séquence (1-34) de la PTH humaine dans les dosages biologiques in vitro (activation de l'adényl-cyclase des cellules rénales) ou in vivo (hypercalcémie induite) (Burtis 1992).

Cependant, les formes circulantes sont encore inconnues, chez l'homme comme chez l'animal. Dans les conditions physiologiques normales, les concentrations plasmatiques sont très faibles et difficilement mesurables par dosage radioimmunologique (RIA). Chez la brebis gestante, elles sont généralement plus élevées dans le plasma fotal que dans le plasma maternel (Ratcliffe et al 1993).

Le peptide joue un rôle fondamental dans la régulation du métabolisme calcique de la femelle gestante et/ou allaitante et dans celle de la croissance foetale et néonatale.

\section{2 / PTHrP et croissance fotale}

Bien que l'existence du PTHrP ait pu être démontrée seulement grâce au syndrome d'hypercalcémie humorale maligne, le gène codant pour ce peptide s'exprime normalement au niveau d'un grand nombre de tissus tels que la peau (kératinocytes), la moelle osseuse, le foie, la muqueuse gastrique, les cellules musculaires lisses gastro-intestinales, utérines et vasculaires. Ce gène s'exprime également au niveau des parathyroïdes fœtales, et PTHrP pourrait être une véritable "PTH fœetale " intervenant en particulier dans la régulation des transferts calciques placentaires.

Figure 1. Schéma des gènes de la PTH et du PTHrP humain (d'après Martin et al 1992). Le gène du $P T H r P$ comprend 9 exons (indiqués par les chiffres romains de là IX), qui peuvent subir un épissage alternatif (indiqué par les traits gris sous chaque schéma). Les exons ou parties d'exons codants ou non codants sont représentés respectivement en noir et en blanc. Le nombre d'acides aminés des différents peptides formés après épissage alternatif en 3' est indiqué à la partie inférieure du schéma.
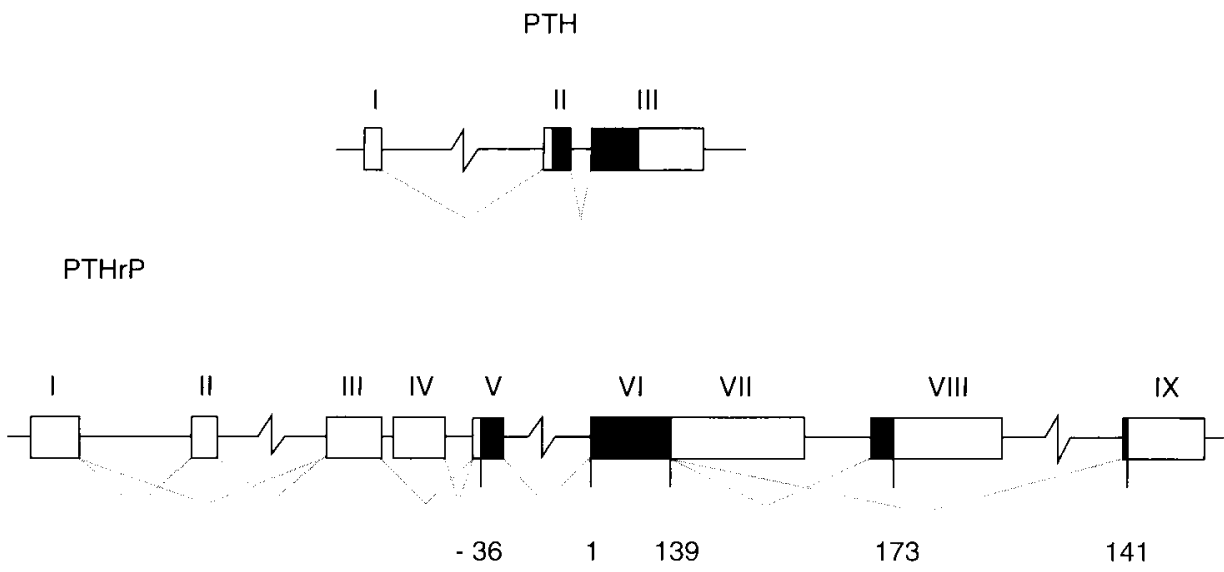


\section{1 / PTHrP et transferts calciques placentaires}

Les ruminants, et en particulier la brebis, constituent un modèle très intéressant pour l'étude des transferts placentaires de calcium. En effet, chez ceux-ci qui possèdent un placenta mésochorial, les transferts calciques placentaires peuvent être considérés comme unidirectionnels dans le sens mère-foetus, la quantité de calcium revenant dans le sens foetus-mère représentant moins de $1 \%$ de celle transférée dans l'autre sens (Braithwaite et al 1970).

Chez les ruminants, comme chez les autres mammifères, il existe un gradient calcique materno-fœetal positif. Bien que très actives pendant la gestation, les parathyroïdes maternelles ne sont pas responsables de ce gradient puisqu'il n'est pas modifié par leur ablation. Par contre, il disparaît après parathyroïdectomie fotale. Il ne peut être que très partiellement restauré par injection de 1,25-dihydroxycalciférol et/ou de PTH au foetus, mais il l'est totalement après administration à celui-ci d'un extrait de ses parathyroïdes. Les concentrations plasmatiques fotales de PTH sont pratiquement indétectables par RIA, alors qu'elles sont supérieures aux concentrations maternelles lorsqu'elles sont évaluées par dosage biologique (cf revues de Garel 1987, Care 1991, Silve 1991). L'ensemble de ces résultats indique qu'un facteur ayant des effets biologiques analogues à ceux de PTH, dont il diffère du point de vue immunologique, est présent dans les parathyroïdes fœtales.

Les parathyroïdes du foetus ovin sécrètent un peptide ayant une activité biologique analogue à celle de $\mathrm{PTH}$, mais des propriétés immunologiques différentes (Rodda et al 1988). Dans les parathyroïdes fotales ovines et bovines, comme dans les membranes placentaires ovines, bovines, porcines et humaines, le dosage RIA a confirmé la présence d'une molécule immunologiquement identique au PTHrP extrait des cellules tumorales (Abbas et al 1990).

Selon Care et al (1990) la partie médiane de PTHrP serait responsable de la stimulation du transfert calcique placentaire, mesuré par perfusion in situ du placenta ovin après enlèvement du fœetus préalablement parathyroïdectomisé. Par ordre croissant, les fragments $(75-84),(75-86)$ et $(67-86)$ du peptide synthétique humain seraient les plus actifs. Ils agiraient sur un récepteur placentaire particulier, différent du récepteur $\mathrm{PTH} / \mathrm{PTH} \mathrm{TP}$ dont le gène s'exprime pourtant au niveau placentaire (Urena et al 1993). Nous avons cependant démontré (Barlet et al 1990) que le fragment synthétique (1-34) de l'hormone humaine pouvait stimuler directement et/ou indirectement (par stimulation de la synthèse de 1,25-dihydroxycholécalciférol) les transferts calciques placentaires chez la brebis gestante (tableau 1). Cet effet peut même s'observer avec de très faibles doses d'hormone, ne modifiant ni la calcémie ni la phosphatémie foetale (Barlet et al 1992b). Les différences entre nos résultats et ceux de Care et al (1990) s'expliquent, au moins partiellement, par la dissemblance des protocoles expérimentaux : perfusion pendant quelques heures du placenta in situ pour ce dernier, injection d'hormone trois fois par jour pendant 10 jours au fœtus cathétérisé chroniquement in utero en ce qui nous concerne.

L'effet du PTHrP sur les transferts de calcium ne semble d'ailleurs pas se limiter au placenta : chez le poulet, le gène de ce peptide s'exprime au niveau des membranes vitelline, amniotique et allantoïdienne, impliquées dans les transports de minéraux vers l'embryon (Schermer et al 1991). L'importance du rôle de cette hormone dans la régulation de ces transferts calciques placentaires a été récemment mise en évidence par Kronenberg (1993) qui, en inactivant le gène codant pour PTHrP chez l'embryon de souris, provoque la mort de tous les nouveau-nés, mort occasionnée par des difficultés respiratoires résultant des déformations thoraciques ainsi induites.

Nous n'avons pas réussi à mettre en évidence un effet du peptide sur les transferts placentaires de phosphate chez la brebis (Barlet et al 1994). Mais le rôle de PTHrP dans la régulation de la croissance foetale ne se limite pas à ses effets au niveau du placenta : PTHrP agit également au niveau de la musculature utérine.

\section{2 / PTHrP et tonicité utérine}

Comme PTH (Pang et al 1981), PTHrP inhibe les contractions utérines induites expérimentalement in vitro (Shew et al 1991, Dalle et al 1992). Les ARNms du PTHrP sont présents dans les cellules musculaires lisses utérines de la ratte gestante, où leur quantité en fin de gestation est proportionnelle à la taille de la portée (Thiede et al 1990). La tension induite au niveau du myomètre par la présence des fotus semble réguler l'expression des ARNms, puisque l'introduction d'un ballonnet plus ou moins gonflé dans la corne utérine d'une ratte gestante chez laquelle les fœetus sont seulement présents dans l'autre corne, induit l'apparition des ARNms dans la

Tableau 1. Influence du fragment (1-34) du PTHrP synthétique humain sur les transferts placentaires de calcium mesurés chez l'agneau du $111^{\circ}$ au $118^{\circ}$ jour de gestation (d'après Barlet et al 1990).

\begin{tabular}{|l|c|c|}
\hline Traitement & $\begin{array}{c}\text { PTHrP } \\
(\mathbf{n = 4})\end{array}$ & $\begin{array}{c}\text { Témoins } \\
(\mathbf{n}=\mathbf{4})\end{array}$ \\
\hline $\begin{array}{l}\text { Poids foetal }(\mathrm{kg}) \\
\begin{array}{l}\text { Transferts calciques } \\
\text { (mmol/24 } \mathrm{h} / \mathrm{kg} \text { de foetus) }\end{array}\end{array}$ & $1,7 \pm 0,2$ & $1,8 \pm 0,1$ \\
\hline
\end{tabular}

(Moyennes \pm écart-type ; ${ }^{*} \mathrm{P}<0,05$, comparaison avec les témoins). Chaque fœtus, cathétérisé chroniquement in utero, recevait quotidiennement par voie jugulaire, du 105" au $117^{*}$ jour de gestation, 6 nmoles d'hormone ou le même volume de solvant. 
première, proportionnellement à la pression de gonflage. Il en est de même si le ballonnet est placé dans l'utérus d'une femelle vierge (Daifotis et al 1992). L'ovariectomie de la ratte réduit considérablement l'expression des ARNms utérins du PTHrP, l'injection d'œstradiol (4 à $400 \mathrm{mg} / \mathrm{kg}$ de poids vif) les multiplie par un facteur de 6 à 8, deux heures après traitement (Paspaliaris et al 1992). Dans l'espèce humaine, les $\mathrm{ARNms}$ du $\mathrm{PTHrP}$ ont également été identifiés dans le myomètre, le placenta, le chorion et l'amnios. Les concentrations du peptide mesurées dans le liquide amniotique $(21 \pm 6 \mathrm{pM}$ à 16 semaines et $41 \pm 9 \mathrm{pM}$ à 38 semaines) sont supérieures à celles mesurées chez les patients souffrant d'hypercalcémie humorale maligne. Elles s'effondrent à l'accouchement, au moment de la rupture des enveloppes, mettant ainsi en évidence un rôle probable du peptide dans le maintien de la quiescence utérine au cours de la grossesse et dans l'expulsion du fœtus au cours du travail (Ferguson et al 1992).

Ces effets du PTHrP aux niveaux placentaire et utérin se retrouvent au niveau de la glande coquillère et des cellules utérines au cours du cycle de formation de l'œuf chez la poule (Thiede et al 1991).

Ainsi, la distension utérine produite par la croissance du (ou des) fœtus augmenterait la production de PTHrP qui agirait localement (par voie autocrine/paracrine) pour induire un relâchement de la musculature utérine permettant au fœetus de se développer normalement.

\section{3 / PTHrP et débit sanguin ombilical}

La tension des cellules musculaires lisses des parois artérielles varie en fonction des changements de pression intravasculaire, de telle sorte que le flux artériel reste relativement constant malgré les modifications locales de pression. Ces mécanismes d'autorégulation font intervenir la réponse contractile de la cellule musculaire lisse à l'étirement. Celui-ci provoque la dépolarisation cellulaire, induisant l'activation des canaux calciques voltage-dépendants. L'influx calcique déclenché par ces derniers provoque une augmentation de la concentration intracellulaire des ions $\mathrm{Ca}^{2+}$, qui se fixent sur une sous-unité protéique, la calmoduline, qui fait partie d'un système enzymatique plus complexe, la MLCK (Myosin Light Chain Kinase). Ce système enzymatique, activé par les ions $\mathrm{Ca}^{2+}$, permet la phosphorylation de la myosine qui, en présence d'actine, d'ions $\mathrm{Mg}^{2+}$ et d'ATP, est alors capable de se fixer sur l'actine et d'hydrolyser l'ATP. Cette hydrolyse fournit l'énergie nécessaire à la déformation des filaments de myosine. Lorsque la concen-

Figure 2. Principaux systèmes associés à un récepteur hormonal membranaire régulant la contraction de la cellule musculaire lisse (d'après Fréminet et al 1993).

Pi : phosphate, PIP2 : phosphoinositol diphosphate, IP3 : inositol triphosphate, DAG : diacylglycérol, CaM : calmoduline, ATP : adénosine triphosphate, ADP : adénosine diphosphate, $M L C K$ : myosin light chain kinase, MLCP : myosin light chain phosphatase.

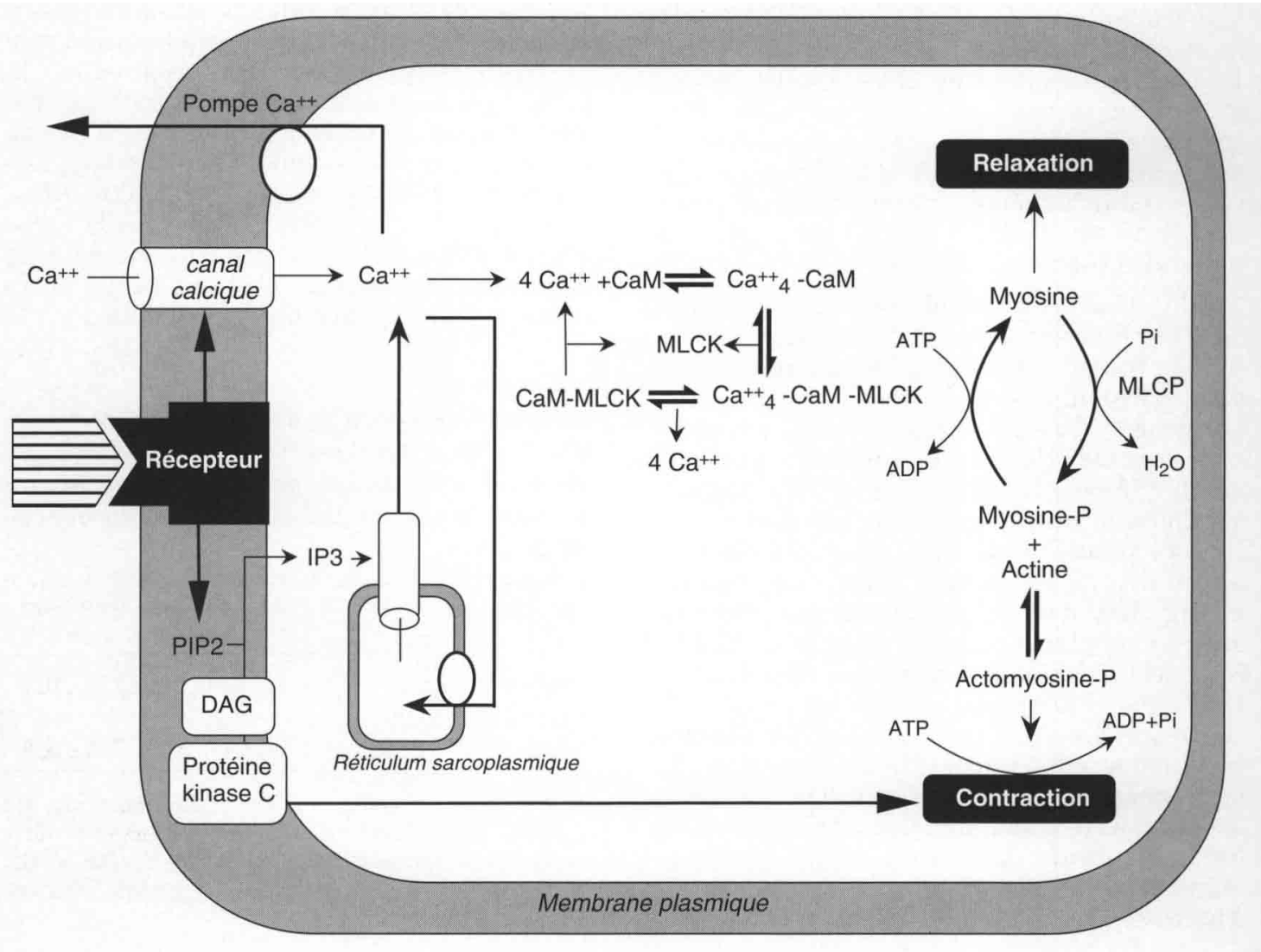


tration intracellulaire des ions $\mathrm{Ca}^{2+}$ est inférieure à $10^{-7} \mathrm{M}$, la $\mathrm{MLCK}$ devient inactive et un autre système enzymatique, la MLCP (Myosin Light Chain Phosphatase), indépendant de $\mathrm{Ca}^{2+}$ et toujours actif, supprime les groupements phosphates des chaînes légères de myosine. En présence d'ATP la tête de myosine se détache des filaments d'actine. Le cycle excitation-contraction s'interrompt et provoque le relâchement musculaire (figure 2) (Fréminet et al 1993).

Il a été démontré depuis longtemps que PTH était dotée de propriétés hypotensives (Charbon et al 1968), indépendantes de son effet hypercalcémiant (Pang et al 1980). L'ARNm de PTHrP s'exprime dans les cellules musculaires lisses des vaisseaux sanguins (Thiede et al 1992), et cette expression est potentialisée par tout étirement subi par la cellule, ou par les vasoconstricteurs tels que l'angiotensine (Pirola et al 1993). PTHrP exerce également un effet vasorelaxant au niveau des artères rénales (Winquist et al 1987), en stimulant la synthèse de monoxyde d'azote à partir de l'arginine (Simeoni et al 1994). Le peptide est produit par les cellules endothéliales des veines ombilicales humaines au niveau desquelles ne s'exprime pas l'ARNm du récepteur PTH/PTHrP qui, en revanche, est présent au niveau des cellules musculaires lisses de ces vaisseaux. Le PTHrP synthétisé par les cellules vasculaires ombilicales peut donc ainsi favoriser la croissance du système vasculaire fœeto-placentaire (Rian et al 1994). Il peut également contrôler le débit sanguin ombilical en modulant les effets vasoconstricteurs de l'endothéline au niveau des artères et des veines ombilicales (Ferguson et al 1994). La résultante de ces effets va donc être une augmentation du débit sanguin irriguant l'unité fœeto-placentaire.

Ainsi, en régulant les transferts calciques placentaires, la tonicité du muscle lisse utérin et le débit sanguin ombilical, PTHrP semble jouer un rôle très important dans la régulation de la croissance fotale. Mais les effets du PTHrP "facteur de croissance » ne se limitent pas à la période fotale. Ils s'étendent également à la période néonatale, car ce peptide intervient aussi dans la régulation du fonctionnement de la glande mammaire dont la production laitière, dans les conditions physiologiques naturelles, conditionne la croissance du nouveau-né.

\section{3 / PTHrP et croissance néonatale}

Les effets vasorelaxants du PTHrP ont été démontrés au niveau pulmonaire (Crass et Scarpace 1993), rénal (Simeoni et al 1994) et ombilical (Ferguson et al 1994). Ils ne sont pas restreints à ces trois territoires et se manifestent également au niveau des artères mammaires.

\section{1 / Production de PTHrP par la glande mammaire}

Thiede et Rodan (1988) ont démontré que l'ARNm du PTHrP s'exprime au niveau de la glande mammaire de la ratte en lactation. $\mathrm{La}$ tétée, en induisant une élévation de la prolactinémie, augmente les ARNms tissulaires du PTHrP. En effet, une injection de prolactine à la ratte en lactation produit les mêmes effets que la tétée, effets inhibés par la bromocriptine (Thiede 1989). Les cellules épithéliales mammaires humaines (Sebag et al 1994) et bovines (Rosol et al 1994) cultivées in vitro produisent du PTHrP. IGF-1 et EGF (Epidermal Growth Factor) stimulent cette production, qui est freinée par la cortisone ou le 1,25-dihydroxycholécalciférol.

Budayr et al (1989) et Thurston et al (1990) furent les premiers à mettre en évidence les concentrations élevées en PTHrP dans le lait (nanomolaires) par rapport à celles (picomolaires) dans le plasma sanguin, aussi bien dans l'espèce humaine que chez le porc, le rat et chez divers ruminants (vache, chèvre, bufflonne). Ceci démontre qu'une partie importante du PTHrP produit par la glande mammaire passe dans le lait, grâce auquel il peut arriver jusqu'au nouveau-né (Goff et al 1991, Ratcliffe et al 1993).

Néanmoins, chez la chèvre (Ratcliffe et al 1992) comme chez la vache (Barlet et al 1993), au moment de la traite ou de la tétée, les concentrations de PTHrP dans les veines mammaires augmentent considérablement, ainsi que le gradient hormonal entre veines et artères mammaires, démontrant ainsi qu'une partie du PTHrP produit par la glande mammaire passe dans le sang et pourra alors agir par voie systémique. De plus, PTHrP stimule la production d'AMPc par les cellules myoépithéliales mammaires (Sebag et al 1994) : il pourrait donc participer au contrôle du stockage et de l'éjection du lait (Ferrari et al 1993).

\section{2 / PTHrP et débit artériel mammaire}

L'ARNm du PTHrP s'exprime au niveau de la paroi de l'artère inguinale de la ratte en lactation, et cette expression est stimulée par la tétée, ou par l'injection de prolactine chez la femelle non lactante (Thiede et al 1992).

Chez la brebis et chez la chèvre, l'injection de PTHrP dans une artère mammaire en augmente le débit (Davicco et al 1993, Thompson 1993, Prosser et al 1994). L'endothéline humaine diminue celui-ci, mais cette diminution peut être complètement inhibée par le PTHrP (Davicco et al 1993, figure 3). Il pourrait ne s'agir que d'un effet pharmacologique puisque les doses de PTHrP nécessaires pour l'observer chez la chèvre correspondent à des concentrations artérielles de $40 \mathrm{pmol} /$, alors que celles mesurées 7 jours après parturition dans une veine mammaire ne dépassent pas 1,2 pmol/l. Cependant, les concentrations du lait en PTHrP, de l'ordre de $8 \times 10^{-9} \mathrm{M}$ 
Figure 3. Influence de l'endothéline perfusée seule (10 pmoles/kg PV; trait noir) ou avec le PTHrP (1-86) humain (100 pmoles/kg PV; trait rouge) sur le débit d'une artère mammaire de la brebis (d'après Davicco et al 1993).

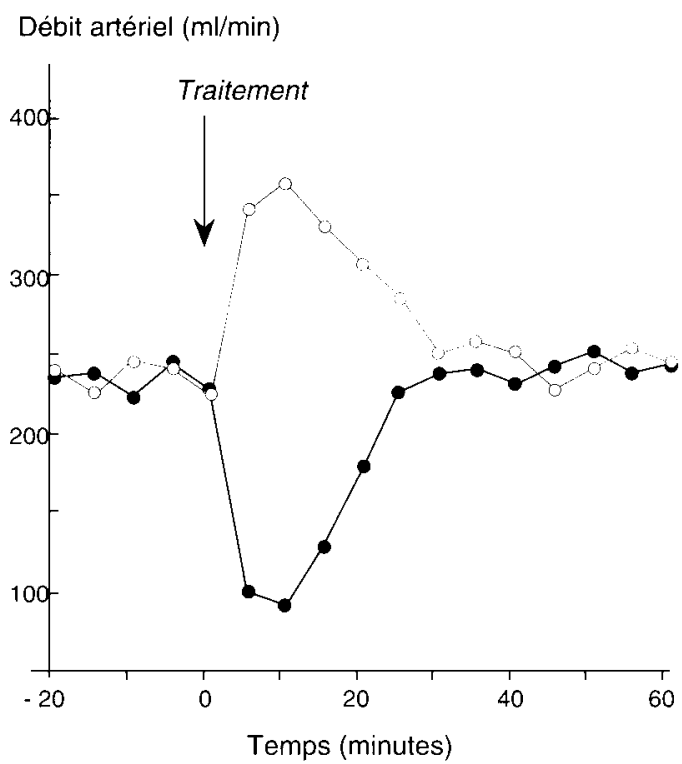

chez cet animal, sont suffisantes pour exercer un effet vasodilatateur (Thompson 1993).

Cet effet vasodilatateur du PTHrP au niveau des artères mammaires pourrait d'ailleurs expliquer partiellement l'influence de ce peptide sur la teneur du lait en minéraux.

\section{3 / PTHrP et minéraux du lait}

PTHrP est impliqué à deux niveaux dans la teneur du lait en calcium et en phosphore. D'une part il contrôle la résorption osseuse observée chez la femelle en lactation. D'autre part, il stimule l'excrétion de ces minéraux au niveau de la mamelle.

\section{a / PTHrP et résorption osseuse chez la femelle en lactation}

Pendant la lactation, chez les ruminants domestiques comme chez la ratte, l'augmentation de l'absorption calcique intestinale est insuffisante pour fournir la totalité du calcium excrété au niveau de la glande mammaire : une fraction plus ou moins importante de celui-ci provient alors du squelette (cf revue Garel 1987). Cette résorption osseuse associée à la lactation (Braithwaite $e t$ al 1970), qui survient également chez l'animal parathyroïdectomisé (Garner et al 1990, Hodnett et al 1992) ne dépend pas de PTH. Brommage et De Luca (1985) avaient émis l'hypothèse selon laquelle le peptide produit au cours de l'hypercalcémie humorale maligne pourrait être normalement produit par la glande mammaire, et serait alors responsable de cette résorption accrue. Les mécanismes d'action du PTHrP au niveau squelettique ont été décrits (cf revue de Rouffet et Barlet 1995).

Bien que l'injection s. c. d'anticorps antiPTHrP humain à des souris allaitantes n'ait eu d'influence ni sur la teneur de leur lait en minéraux ni sur la régulation de leur métabolisme phospho-calcique (Melton et al 1990), chez la ratte, les concentrations plasmatiques de PTHrP s'élèvent au moment du déclenchement de la lactation (Miller et al 1991), lorsque la résorption osseuse est maximale (Miller 1991).

Ainsi, les minéraux provenant de la résorption osseuse accélérée, et de l'absorption intestinale, sont véhiculés au niveau de la mamelle, où PTHrP intervient également pour moduler leur excrétion.

\section{b / PTHrP et teneur du lait en calcium et en phosphore}

Les études réalisées ont montré qu'il existait chez les bovins une corrélation positive entre les teneurs du lait en calcium et en PTHrP. Ainsi, le lait des vaches Jersiaises, plus riche en calcium que celui des Holstein, présente également une teneur supérieure en PTHrP (Law et al 1991). Cette corrélation ne se retrouve toutefois pas chez la ratte (Yamamoto et al 1992), chez qui la teneur du lait en PTHrP augmente au cours de la lactation alors que celle du calcium reste constante (Rakopoulos et al 1992).

PTHrP stimulant les transferts de calcium au niveau du placenta (Barlet et al 1990), nous avons étudié son éventuel effet sur ces transferts au niveau de la mamelle : les fragments synthétiques (1-34) et (1-86) du PTHrP humain, perfusés par voie intraveineuse à la chèvre en début de lactation, augmentent la teneur du lait en calcium et en phosphore (Barlet et al 1992a).

Stimulant les transferts de calcium successivement au niveau placentaire et mammaire, le PTHrP pourrait donc favoriser la nutrition minérale du nouveau-né, comme il le fait pour celle du fœetus. Mais, pour véritablement optimiser la nutrition phospho-calcique du nouveau-né, celui-ci doit être en outre capable d'utiliser convenablement les minéraux qui lui sont apportés. Le PTHrP fourni par le lait pourrait donc également exercer un contrôle sur la motricité gastrointestinale et sur l'absorption du calcium et du phosphore.

\section{4 / PTHrp et tractus digestif du nouveau-né}

\section{a / Motricité digestive}

Les effets du PTHrP concernant la cellule musculaire lisse s'exercent non seulement au niveau de la paroi des vaisseaux sanguins ou de l'utérus, mais également au niveau des cellules de la paroi gastro-intestinale.

PTHrP provoque le relâchement des parois gastriques et intestinales du porc et du rat 
(Mok et al 1989). Alors que les fragments (1-16) ou (7-34) du PTHrP synthétique humain sont dépourvus d'effet, le fragment (1-34) inhibe les contractions induites par la cholécystokinine sur des cellules isolées de l'intestin de cobaye. Cette inhibition ne se manifeste pas sur des cellules prétraitées soit par un inhibiteur compétitif spécifique du récepteur $\mathrm{PTH} / \mathrm{PTH} \mathrm{P}$ ou du récepteur du peptide intestinal vasoactif (VIP), soit par la somatostatine ou la N-éthylmaléimide (inhibant l'adényl-cyclase), soit par le (R)-p-AMPc (antagoniste de l'AMPc au niveau de la protéine kinase A). Ceci démontre que le PTHrP provoque le relâchement observé par un effet direct au niveau de la cellule lisse, faisant intervenir le récepteur du VIP, l'adénylcyclase et la protéine kinase A (Botella et al 1994).

Mais, outre le contrôle de la motricité gastro-intestinale, le PTHrP pourrait également intervenir sur l'absorption intestinale des minéraux.

\section{b / Absorption calcique}

PTHrP induit l'apparition d'une transcaltachie (stimulation rapide et transitoire de l'absorption calcique intestinale) sur une anse ligaturée d'intestin de poulet. Cet effet peut être bloqué par la nifédipine (inhibiteur des canaux calciques) (Zhou et al 1992). Chez le mouton adulte, PTHrP stimule l'absorption de calcium et de phosphore au niveau de l'épithélium ruminal (Dua et al 1994). L'injection d'un sérum anti-PTHrP humain à des souriceaux nouveau-nés ne modifie cependant pas les paramètres de leur métabolisme phospho-calcique (Kukreja et al 1991). De même, chez des porcelets de 2 jours, la supplémentation en PTHrP de leur aliment d'allaitement à base de lait de soja (donc totalement dépourvu de PTHrP), destiné aux nouveau-nés humains, ne fait pas varier significativement l'absorption calcique intestinale (Hillman et al 1994).

Ces deux expérimentations ne permettent pourtant pas d'éliminer un éventuel rôle du peptide sur ce dernier paramètre. En effet, dans l'expérience conduite chez les souriceaux, l'anticorps était dirigé contre l'hormone humaine. De plus, une injection intraveineuse de cet anticorps, même actif contre l'hormone murine, n'inhiberait pas les effets autocrines/paracrines du peptide apporté par le lait. Dans la deuxième expérience, l'aliment distribué aux porcelets avait une composition analogue à celle du lait humain ; il était beaucoup moins riche en calcium $(0,3$ us $2,2 \mathrm{~g} / \mathrm{l})$ et en phosphore $(0,16$ vs $1,55 \mathrm{~g} / \mathrm{l})$ que le lait de truie. De plus, chez le veau nouveau-né de un jour, l'addition de PTHrP (1-34) synthétique humain à un lait de soja (1nmole de peptide par litre) accélère la vitesse de passage dans le sang du xylose (marqueur de l'absorption intestinale) ajouté à ce lait, la rapprochant de celle du xylose ajouté au colostrum (figure 4 ).
Si la bonne utilisation du calcium alimentaire est une condition nécessaire à la croissance néonatale, celle-ci est régulée par les somatomédines, et en particulier par l'IGF-1 (Chevenne 1991).

\section{5 / PTHrP et synthèse d'IGF-1 par l'os et le foie}

Les IGFs régulent le métabolisme et la prolifération des ostéoblastes (McCarthy et al 1989a). PTH stimule la synthèse ostéoblastique d'IGF-1, ce qui explique l'effet anabolisant de cette hormone au niveau osseux (McCarthy et al 1989b). Les effets de PTHrP au niveau ostéoblastique sont analogues à ceux de PTH : il stimule la synthèse de collagène et d'IGF-1 (Canalis et al 1990).

Chez le jeune veau préruminant, nous avons démontré qu'une perfusion de PTHrP dans la veine porte stimulait la production d'IGF-1 par le tissu hépatique (Coxam et al 1992), au niveau duquel s'exprime l'ARNm du récepteur PTH/PTHrP (Urena et al 1993). PTHrP peut donc stimuler la croissance osseuse néonatale selon un double processus autocrine/paracrine et endocrine, augmentant la production d'IGF-1 respectivement au niveau osseux et au niveau hépatique.

Figure 4. Concentrations plasmatiques de xylose mesurées chez 3 veaux nouveau-nés après ingestion d'un repas de colostrum ou de lait de soja supplémenté (1 nmole/l) ou non en PTHrP (1-34) humain.

Le lait de soja était additionné de protéines, lipides, lactose et minéraux, de façon à ce que sa composition soit analogue à celle du colostrum bovin (Davicco et Barlet, données non publiées).

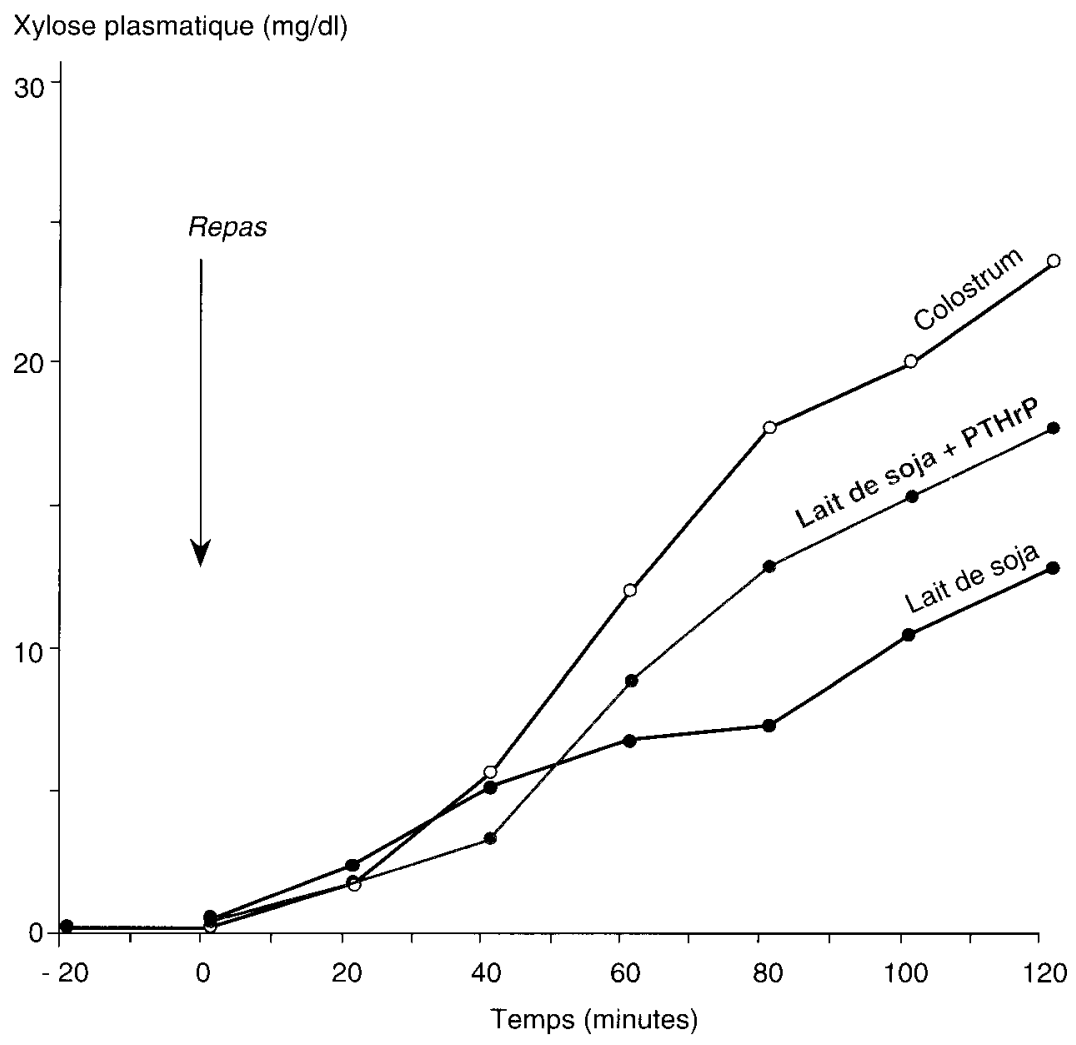


Tableau 2. Récapitulatif des effets actuellement connus du PTHrP sur la croissance fœtale et néonatale.

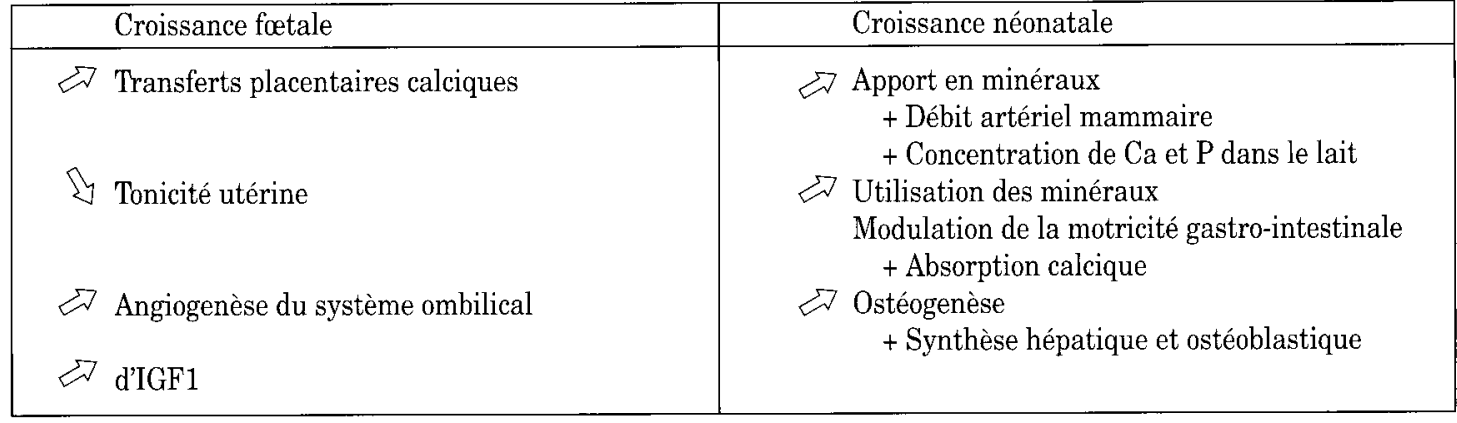

\section{Conclusion}

Le PTHrP joue un rôle très important dans la régulation du métabolisme calcique au cours de la gestation et de la lactation, et dans celle de la croissance osseuse chez le fœtus et le nouveau-né (tableau 2). Chez la femelle gestante, le peptide élaboré par les parathyroïdes fotales et les membranes placentaires stimule les transferts calciques de la mère vers le fœtus, et favorise ainsi la croissance du squelette de ce dernier. Cette croissance s'effectue dans des conditions optimales grâce à l'effet relaxant exercé par le peptide au niveau de la musculature utérine.

Chez la femelle allaitante, le PTHrP sécrété par la glande mammaire stimule la résorption osseuse indispensable à la fourniture du calcium et du phosphore exportés par la mamelle. Il participe également à la régu- lation de la teneur du lait en ces minéraux, peut-être en modulant l'effet vasoconstricteur de l'endothéline au niveau des artères mammaires. Chez le nouveau-né, outre un éventuel effet indirect sur la croissance osseuse faisant intervenir un contrôle de la motricité gastro-intestinale et de l'absorption calcique intestinale, il intervient aussi directement sur cette croissance en stimulant la synthèse d'IGF-1 au niveau du foie et de l'os.

Le PTHrP apparaît donc aujourd'hui comme un régulateur du métabolisme phospho-calcique au cours de la gestation et de la lactation. Ce rôle pourrait être particulièrement important chez la femelle des ruminants domestiques, souvent simultanément gestante et allaitante. Il l'est tout autant en ce qui concerne la croissance osseuse fœtale et néonatale.

\section{Références bibliographiques}

Abbas S. K., Pickard D. W., Illingworth D., Store J., Purdie D. W., Moniz C., Dixit M., Caple I. W., Ebeling P. R., Rodda C. P., Martin T. J., Care A. D. 1990. Measurement of parathyroid hormone-related protein in extracts of fetal parathyroid glands and placental membranes. J. Endocrinol., 124, 319-325.

Barlet J.-P., 1974. Rôle physiologique de la calcitonine chez la chèvre gestante ou allaitante. Ann. Biol. anim. Bioch. Biophys., 14, 447-458.

Barlet J.-P., Davicco M.-J., Coxam V., 1990. Synthetic parathyroid hormone-related peptide (1-34) fragment stimulates placental calcium transfer in ewes. J. Endocrinol., 127, 33-37.

Barlet J.-P., Champredon C., Coxam V., Davicco M.-J., Tressol J.-C., 1992a. Parathyroid hormonerelated peptide might stimulate calcium secretion into the milk of goats. J. Endocrinol., 132, 353-359.

Barlet J.-P., Davicco M.-J., Coxam V., 1992b. Calcitonin modulates parathyroid hormone-related peptide-stimulated calcium placental transfer. In : D. V. Cohn, C. Gennari, A. H. Tashjian (eds.), Calcium regulating hormones and bone metabolism, 124-128. Elsevier, Amsterdam.

Barlet J.-P., Abbas S. K., Care A. D., Davicco M.-J., Rouffet J.,1993. Parathyroid hormone-related pep- tide and milking-induced phosphaturia in dairy cows. Acta Endocrinol., 129, 332-336.

Barlet J.-P., Davicco M.-J., Rouffet J., Coxam V., Lefaivre J., 1994. Parathyroid hormone-related peptide dœs not stimulate phosphate placental transport. Placenta, 15, 441-444.

Botella A., Rekik M., Delvaux M., Davicco M.-J., Barlet J.-P., Frexinos J., Bueno L., 1994. Parathyroid hormone (PTH) and PTH-related peptide induce relaxation of smooth muscle cells from guinea pig ileum : interaction with vasoactive intestinal peptide receptors. Endocrinology, 135, 21602167.

Braithwaite G. D., Glascock R. F., Riazuddin S. H., 1970. Calcium metabolism in pregnant ewes. $\mathrm{Br}$. J. Nutr., 24, 661-670.

Broadus A. E., Mangin M., Ikeda K., Insogna K. L., Weir E. C., Burtis W. J., Stewart A. F., 1988. Humoral hypercalcemia of cancer. Identification of a novel parathyroid hormone-like peptide. N. Engl. J. Med., 319, 556-563.

Brommage R., De Luca H. F., 1985. Regulation of bone mineral loss during lactation. Am. J. Physiol., 248, E182-E187. 
Budayr A. A., Halloran B. P., King J. C., Diep D., Nissenson R. A., Strewler G. J., 1989. High levels of parathyroid hormone-like protein in milk. Proc. Natl. Acad. Sci. USA, 86, 7183-7185.

Burtis W. J., 1992. Parathyroid hormone-related protein : structure, function and measurement. Clin. Chem., 38, 2171-2183.

Canalis E., McCarthy T. L., Centrella M., 1990. Differential effects of continuous and transient treatment with parathyroid hormone-related peptide (PTHrP) on bone collagen synthesis. Endocrinology, $126,1806-1812$.

Care A. D., 1991. The placental transfer of calcium. J. Develop. Physiol., 15, 253-257.

Care A. D., Abbas S. K., Pickard D. W., Barri M., Drinkhill M., Findlay J. B. C., White I. R., Caple I. W., 1990. Stimulation of ovine placental transport of calcium and magnesium by mid-molecule fragments of human parathyroid hormone-related protein. Exp. Physiol., 75, 605-608.

Charbon G. A., Brummer F., Reneman R. S., 1968. Diuretic and vascular action of parathyroid extracts in animals and man. Arch. int. Pharmacodyn., 171, 1-11.

Chevenne D., 1991. Les somatomédines. Ann. Biol. Clin., 49, 69-91.

Coxam V., Davicco M.-J., Durand D., Lefaivre J., Barlet J.P., 1992. The influence of parathyroid hormone-related peptide on hepatic IGF-1 production. Acta Endocrinol., 126, 430-433.

Crass M. F., Scarpace P. J., 1993. Vasoactive properties of a parathyroid hormone-related protein on rat aorta. Peptides, 14, 179-183.

Daifotis A. G., Weir E. C., Dreyer B. A., Broadus A. E., 1992. Stretch-induced parathyroid hormonerelated peptide gene expression in the rat uterus. J. Biol. Chem., 267, 23455-23458.

Dalle M., Dauprat-Dalle P., Barlet J.-P., 1992. Parathyroid hormone-related peptide inhibits oxytocininduced rat uterine contractions in vitro. Arch. Int. Physiol. Bioch. Biophys., 101, 251-254.

Davicco M.-J., Rouffet J., Durand D., Lefaivre J., Barlet J.-P., 1993. Parathyroid hormone-related peptide might increase mammary blood flow. J. Bone Miner. Res., 8, 1519-1524.

Dua K., Leonhard S., Martens H., Abbas S. K., Care A. D., 1994. Effects of parathyroid hormone and parathyroid hormone-related protein on the rates of absorption of magnesium, calcium, sodium, potassium and phosphate ions from the reticulo-rumen of the sheep. Exp. Physiol., 79, 401-408.

Ferguson J. E., Gorman J. V., Bruns D. E., Weir E. C., Burtis W. J., Martin T. J., Bruns M. E., 1992. Abundant expression of parathyroid hormone-related protein in human amnion and its association with labor. Proc. Natl. Acad. Sci. USA, 89, 8384 8388 .

Ferguson J. E., Seaner R., Bruns D. E., Redick J. A., Mills S. E., Jüppner H., Segre G. V., Bruns M. E. 1994. Expression of parathyroid hormone-related protein and its receptors in human umbilical cord: evidence for a paracrine system involving umbilical vessels. Am. J. Obstet. Gynecol., 170, 1018-1026.
Ferrari S., Rizzoli R., Chaponnier C., Gabbiani G., Bonjour J.-P., 1993. Parathyroid hormone-related protein increases cAMP production in mammary epithelial cells. Am. J. Physiol., 264, E471-E475.

Fréminet A., Verdetti J., Bourdon J., 1993. Hormones et fonctionnement de l'appareil cardiovasculaire. In : J.-P. Dupouy (ed.), Hormones et Grandes Fonctions, Tome 2, 303-389. Marketing, Paris.

Garel J.-M., 1987. Hormonal control of calcium metabolism during the reproductive cycle in mammals. Physiol. Rev., 67, 1-66.

Garner S. C., Boass A., Toverud S. U., 1990. Parathyroid hormone is not required for normal milk composition or secretion or lactation-associated bone loss in normocalcemic rats. J. Bone Min. Res. 5, 6975.

Goff J.-P., Reinhardt T. A., Lee S., Hollis B. W. 1991. Parathyroid hormone-related peptide content of bovine milk and calf blood assessed by radioimmunoassay and bioassay. Endocrinology, 129, 28152819.

Hillman L. S., Forte L. R., Veum T., Ru Y., Howard B., Rowe A., Hillman H. W., 1994. Effect of parathyroid hormone-related peptide supplementation of soy protein formulas in the neonatal pig model. J. Bone Min. Res., 9, 1047-1052.

Hodnett D. W., De Luca H. F., Jorgensen N. A., 1992. Bone mineral loss during lactation occurs in absence of parathyroid tissue. Am. J. Physiol., 262, E230-E233.

Jüppner H., Abou-Samra A.-B., Freeman M., Kong X. F., Schipani E., Richards J., Kolakowski S. F., Hock J., Potts J. T., Kronenberg H. M., Segre G. V., 1991. A G protein-linked receptor for parathyroid hormone and parathyroid hormone-related peptide. Science, 254, 1024-1026.

Kronenberg H. M., 1993. Effect of the ablation of the PTHrP gene in vivo. Calcif. Tissue Int., 52 (supplt 1), S106.

Kukreja S. C., D'Anza J. J., Melton M. E., Wimbiscus S. A., Grill V., Martin T. J., 1991. Lack of effect of neutralization of parathyroid hormone-related protein on calcium homeostasis in neonatal mice. J. Bone Min. Res., 6, 1197-1201.

Law F. M. K., Moate P. J., Leaver D. D., DiefenbachJagger H., Grill V., Ho P. W. M., Martin T. J., 1991 Parathyroid hormone-related protein in milk and its correlation with bovine milk calcium. J. Endocrinol, $128,21-26$

Martin T. J., Moseley J. M., Gillespie M. T., 1992. Regulation of the PTHrP gene and its protein products. In : D. V. Cohn, C. Gennari, A. H. Tashjian (eds.), Calcium regulating hormones and bone metabolism, 25-35. Elsevier, Amsterdam.

McCarthy T. L., Centrella M., Canalis E., 1989a. Insulin-like growth factor (IGF) and bone. Connect. Tissue Res., 20, 277-282.

McCarthy T. L., Centrella M., Canalis E., 1989b. Parathyroid hormone enhances the transcript and polypeptide of insulin-like growth factor 1 in osteoblast-enriched cultures from fetal rat bone. Endocrinology, 124, 1247-1253.

Melton M. E., D'Anza J. J., Wimbiscus S. A., Grill V., Martin T. J., Kukreja S. C., 1990. Parathyroid 
hormone-related protein and calcium homeostasis in lactating mice. Am. J. Physiol., 259, E792-E796.

Miller S. C., 1991. Regulation of osteoclastic resorption. Calcif. Tissue Int., 48 (supplt), A2.

Miller S. C., Bagi C. M., Bowman B. M., 1991. Increased serum levels of parathyroid hormone-related peptide correlate the activation of bone resorption and remodeling during early lactation in rats. Calcif. Tissue Int., 48 (supplt), A13.

Mok L. L. S., Cooper C. W., Thompson J. C., 1989. Parathyroid hormone and parathyroid hormonerelated protein inhibit phasic contraction of pig duodenal smooth muscle. Proc. Soc. exp. Biol. Med., $192,337-340$

Moseley J. M., Kubota M., Diefenbach-Jagger H., Wettenhall R. E. H., Kemp B. E., Suva L. J., Rodda C. P., Ebeling P. R., Hudson P. J., Zajac J. D., Martin T. J., 1987. Parathyroid hormone-related protein purified from a human lung cancer cell line. Proc. Natl. Acad Sci. USA, 84, 5048-5052.

Munson P. L., 1955. Studies on the role of the parathyroids in calcium and phosphorus metabolism Ann. N. Y. Acad. Sci., 60, 776-796.

Pang P. K. T., Tenner T. E., Yee J. A., Janssen H. F., 1980. Hypotensive action of parathyroid hormone preparations in rats and dogs. Proc. Natl. Acad. Sci USA, 77, 675-678.

Pang P. K. T., Shew R. L., Sawyer W. H., 1981. Inhibition of uterine contraction by synthetic parathyroid hormone fragment. Life Sci., 28, 1317-1321.

Parsons J. A., 1976. Parathyroid physiology and the skeleton. In: G. H. Bourne (ed.), Biochemistry and Physiology of Bone, vol. IV, 159-225. Academic Press, New York.

Paspaliaris V., Vargas S. J., Gillespie M. T. Williams E. D., Danks J. A., Moseley J. M., Story M. E., Pennefather J. M., Leaver D. D., Martin T. J. 1992. Oestrogen enhancement of the myometrial response to exogenous parathyroid hormone-related protein (PTHrP) and tissue localization of endogenous PTHrP and its mRNA in the virgin rat uterus J. Endocrinol., 134, 415-425.

Pirola C. J., Wang H.-M., Kamyar A., Wu S., Enomoto H., Sharifi B., Forrester J. S., Clemens T. L., Fagin J. A., 1993. Angiotensin II regulates parathyroid hormone-related protein expression in cultured rat aortic smooth muscle cells through transcriptional and post-transcriptional mechanisms. J. Biol. Chem., 268, 1987-1994.

Prosser C. G., Farr J. C., Davis S. R., 1994. Increased mammary blood flow in the lactating goat induced by parathyroid hormone-related protein. Exp. Physiol., 79, 565-570.

Rakopoulos M., Vargas S. J., Gillespie M. T., Ho P. W. M., Diefenbach-Jagger H., Leaver D. D., Grill V., Moseley J. M., Danks J. A., Martin T. J., 1992. Production of parathyroid hormone-related protein by the rat mammary gland in pregnancy and lactation. Am. J. Physiol., 263, E1077-E1085.

Ratcliffe W. A., Thompson G. E., Care A. D., Peaker M., 1992. Production of parathyroid hormone-related protein by the mammary gland of the goat. J. Endocrinol., 133, 87-93.

Ratcliffe W. A., Abbas S. K., Care A. D., 1993. Clearance of parathyroid hormone-related protein in pregnant, non pregnant and fetal sheep, goats and pigs. J. Endocrinol., 138, 459-465.

Rian E., Jemtland R., Olstad O. Kr., Endresen M. J., Grasser W. A., Thiede M. A., Henriksen T., Bucht E., Gautwik K. M., 1994. Parathyroid hormone-related protein is produced by cultured endothelial cells : a possible role in angiogenesis. Biochem. Biophys. Res. Commun., 198, 740-747.

Rodda C. P., Kubota M., Heath J. A., Ebeling P. R., Moseley J. M., Care A. D., Caple I. W., Martin T. J. 1988. Evidence for a novel parathyroid hormonerelated protein in fetal lamb parathyroid glands and sheep placenta : comparison with a similar protein implicated in humoral hypercalcaemia of malignancy. J. Endocrinol., 117, 261-271.

Rosol T. J., Capen C. C., 1988. Pathogenesis of humoral hypercalcemia of malignancy. Domest. Anim. Endocrinol., 5, 1-21.

Rosol T. J., Okada H., Schanbacher F. L., McCauley L. K., Weckmann M. T., Capen C. C., 1994. Parathyroid hormone-related protein production in vitro by mammary cells from lactating cows. J. Bone Min. Res., 9 (supplt 1), C414.

Ross R., Care A. D., Robinson J. S., Pickard D. W. Weatherley A. J., 1980. Perinatal 1, 25-dihydroxycholecalciferol in the sheep and its role in the maintenance of the transplacental calcium gradient. J. Endocrinol., 87, 17P-18P.

Rouffet J., Barlet J.-P., 1995. Peptide apparenté à l'hormone parathyroïdienne (PTHrP) et métabolisme osseux. Arch. Physiol. Bioch., 103, 3-13.

Schermer D. T., Chan S. D. H., Bruce R., Nissenson R. A., Wood W. I., Strewler G. J., 1991. Chicken parathyroid hormone-related protein and its expression during embryonic development. J. Bone Min. Res., 6, 149-155.

Sebag M., Henderson J., Goltzman D., Kremer R. 1994. Regulation of parathyroid hormone-related peptide production in normal human mammary epithelial cells in vitro. Am. J. Physiol., 267, C723C730.

Shew R. L., Yee J. A., Kliewer D. B., Keflemarian Y. J., McNeill D., 1991. L., Parathyroid hormonerelated protein inhibits stimulated uterine contractions in vitro. J. Bone Min. Res., 6, 955-959.

Silve C., 1991. Parathyroid hormone related peptide, une nouvelle hormone. Méd. Sci., 7, 52-57.

Simeoni U., Massfelder T., Saussine C., Judes C., Geisert J., Helwig J.-J., 1994. Involvement of nitric oxide in the vasodilatory response to parathyroid hormone-related peptide in the isolated rabbit kidney. Clin. Sci., 86, 245-249.

Thiede M. A., 1989. The mRNA encoding a parathyroid hormone-like protein is produced in mammary tissue in response to elevations in serum prolactin. Mol. Endocrinol., 87, 1443-1447.

Thiede M. A., Rodan G. A., 1988. Expression of a calcium mobilizing parathyroid hormone-like peptide in lactating mammary tissue. Science, 242,278 280.

Thiede M. A., Daifotis A. G., Weir E. C., Brines M. L., Burtis W. J., Ikeda K., Dreyer B. E., Garfield R. E., Broadus A. E., 1990. Intrauterine occupancy controls expression of the parathyroid hormone - 
related peptide gene in preterm rat myometrium. Proc. Natl. Acad Sci. USA, 87, 6969-6973.

Thiede M. A., Harm H. C., Mc Kee R. L., Grasser W. A., Duong L. T., Leach R. M., 1991. Expression of the parathyroid hormone-related protein gene in the avian oviduct : potential role as a local modulator of vascular smooth muscle tension and shell gland motility during the egg-laying cycle. Endocrinology, 129, 1958-1966.

Thiede M. A., Grasser W. A., Petersen D. N., 1992. Regulated expression of parathyroid hormone-related protein in mammary blood supply supports a role in mammary blood flow. In : D. V. Cohn, C. Gennari, A. H. Tashjian (eds.), Calcium regulating hormones and bone metabolism, 62-66. Elsevier, Amsterdam.

Thompson G. E., 1993. Parathyroid hormone-related protein and mammary blood flow in the sheep. Exp. Physiol., 78, 499-501.

Thurston A. W., Cole J. A., Hillman L. S., Im J. H., Thorne P. K., Krause W. J., Jones J. R., Eber S. L., Forte L. R., 1990. Purification and properties of parathyroid hormone-related peptide isolated from milk. Endocrinology, 126, 1183-1190.
Urena P., Kong X.-F., Abou-Samra A.-B., Jüppner H., Kronenberg H. M., Potts J. T., Segre G. V., 1993. Parathyroid hormone (PTH)/PTH-related peptide receptor messenger ribonucleic acids are widely distributed in rat tissues. Endocrinology, 133, 617-623.

Weryha G., Leclère J., 1995. Paracrine regulation of bone remodeling. Horm. Res., 43, 69-75.

Winquist R. J., Baskin E. P., Vlasuk G. P., 1987. Synthetic tumor-derived human hypocalcemic factor exhibits parathyroid hormone-like vasorelaxation in renal arteries. Biochem. Biophys. Res. Commun., 149, 227-232.

Yamamoto M., Fisher J. E., Thiede M. A., Caulfield M. P., Rosenblatt M., Duong L. T., 1992. Concentrations of parathyroid hormone-related protein in rat milk change with duration of lactation and interval from previous suckling, but not with milk calcium. Endocrinology, 130, 741-747.

Zhou L.-X., Nemere I., Norman A. W., 1992. A parathyroid hormone-related peptide induces transcaltachia (the rapid, hormonal stimulation of intestinal $\mathrm{Ca}^{2+}$ transport). Biochem. Biophys. Res. Commun., 186, 69-73.

\section{Bone growth in the fetus and the neonate: role of parathyroid hormone-related peptide.}

Parathyroid hormone-related peptide (PTHrP) plays a major role in the regulation of bone growth in the fetus and the neonate. In pregnant and/or lactating ruminant animals, the PTHrP secreted by fetal parathyroid glands and placen. tal membranes stimulates calcium placental transfer from the dam to the fetus. PTHrP also modulates myometrial uterine activity. The expression of the PTHrP gene in pregnant uterus is under the control of mechanical stretch indu. ced by uterine distension following fetal growth. Thus PTHrP controls the rhytmicity and/or force of myometrial contractions during pregnancy. Since uterine contractions severely depress blood flow, another possibility is that PTHrP might act to dilate uterine (and placental) blood vessels and assure adequate blood flow to the uterus and feto-placental unit. Conversely, in ewes and goats, the PTHrP released by the lactating mammary gland regulates mammary blood flow and calcium and phosphorus mammary secretion. In neonates, milk PTHrP might control gut motility and calcium and phosphorus intestinal absorption.

Thus, by controling calcium placental transfer, myometrial uterine activity, placental and mammary blood flow, milk calcium and phosphorus concentration, and, possibly, calcium and phosphorus intestinal absorption in the newborn, PTHrP regulates skeletal growth during fetal and postnatal periods.

BARLET J.-P., DAVICCO M.-J., COXAM V., 1995. Croissance osseuse au cours des périodes fœtale et néonatale : rôle du peptide apparenté à l'hormone parathyroïdienne. INRA Prod. Anim., 8 (3), 177-187. 